\title{
Solution to Security Constrained Environmental Pumped-Storage Hydraulic Unit Scheduling Problem by Genetic Algorithm
}

\author{
Salih Fadil, Cüneyt Demir, and Burak Urazel \\ Department of Electrical Engineering, Eskişehir Osmangazi University, Meşelik, 26480 Eskişehir, Turkey \\ Correspondence should be addressed to Salih Fadil; sfadil@ogu.edu.tr
}

Received 2 May 2013; Accepted 5 August 2013

Academic Editors: C. Liu and A. Safacas

Copyright (c) 2013 Salih Fadil et al. This is an open access article distributed under the Creative Commons Attribution License, which permits unrestricted use, distribution, and reproduction in any medium, provided the original work is properly cited.

\begin{abstract}
A lossy electric power system area that contains thermal units and a pumped-storage (p-s) hydraulic unit is considered in this paper. The cost function, which is weighted combination of the total fuel cost and the total emission cost of the thermal units, in an operation cycle, is minimized under some possible electric and hydraulic constraints. The dispatch technique that is based on genetic algorithm considers minimum and maximum reservoir storage limits of the p-s unit, upper and lower active and reactive generation limits of the thermal units, upper and lower active pumping/generation power limits of the p-s unit, maximum transmission capacities of the transmission lines, and upper and lower limits of the bus voltage magnitudes in a considered power system. The proposed dispatch technique was tested on an example power system that has 12 buses with five thermal units and a p-s hydraulic unit. The same dispatch problem is solved via an iterative solution method based on modified subgradient algorithm operating on feasible values (F-MSG) and pseudowater price just for comparison purpose. It is seen that the solution technique based on the F-MSG algorithm and pseudowater price gives similar results with the proposed algorithm based on genetic algorithm.
\end{abstract}

\section{Introduction}

The main function of p-s hydraulic units in electric power systems is to store inexpensive surplus electric energy that is available during off-peak load levels as hydraulic potential energy. This is done by pumping water from the lower reservoir of a p-s unit to its upper reservoir. The stored hydraulic potential energy is then used to generate electric energy during peak load levels (peak shaving hydraulic units). P$s$ units are generally operated over daily or weekly periods. Operation of a p-s unit over a period can reduce the total cost in a power system.

In [1], enhanced merit order and augmented Lagrange Hopfield network method for solving hydrothermal scheduling problem with pumped-storage units are used. A complete methodology to define the dimensions of an autonomous electricity generation system based on the maximum available solar energy at minimum electricity generation cost is given in [2]. In this study, the most cost efficient energy storage mean including pumped-storage unit is tried to be found. In [3], the power storage options including pumpedstorage unit is analyzed to support the wind energy integration to a power system. The French power system is taken as the case study in this paper. An optimization method based on mixed linear integer programming is used in solution of large scale hydrothermal scheduling problem with pumpedstorage hydroplant in [4]. Some previous papers about p$s$ unit scheduling problem during nineteen sixties can also be found in [5]. In our previous work, p-s hydraulic unit scheduling problem in a lossy electric power system is solved by using the pseudospot price of electricity algorithm in [6]. In this paper some security constraints, such as bus voltage magnitudes and transmission line maximum transmission capability constraints, and emission costs of the thermal units are not considered. In [7], combined optimization of wind farm and p-s unit in a market environment is investigated. 
The problem is modeled as two-stage stochastic programming problem that considers two random parameters: market price and wind generation. The researchers modeled two different joint operations of the units and compare it with the uncoordinated operation of them. A solution method based on the genetic algorithm, which is similar to the one given in this paper, is applied to the short-term hydrothermal scheduling problem in [8].

In our work, formulation of the optimization problem is divided into two parts as active and reactive power optimization subproblems. In the active power optimization, active generations of all units (except the one connected to the swing bus) in all subintervals are determined by means of the genetic algorithm. The reactive generations of all units (except the one connected to the swing bus) in all subintervals are taken as equal to the predefined values, and they are not changed during the active power optimization. In the reactive power optimization, active generations of all units (except the one connected to the swing bus) in all subintervals are taken as equal to the optimal values that have been found in the active power optimization calculation. Only reactive generations in all subintervals are determined by means of the genetic algorithm. Consequently, at the end of the reactive power optimization, Pareto optimal solutions for the power system have been calculated.

In our solution, active and reactive power generation constraints of the thermal units (except the one connected to swing bus), generation, and pumping active power constraints of the p-s unit are handled via coding and mapping mechanism of the genetic algorithm [9]. Active and reactive power generation constraints of the thermal unit that is connected to swing bus, p-s unit's reservoir constraints, bus voltage magnitude constraints, and transmission lines' maximum transmission capacity constraints are added into the problem via respective penalty terms. The fuel cost rate and gas emission rate curves for each unit are combined by means of weighting factor $w$, which varies between 0 and 1 . Detailed explanation about the fundamental structure of the genetic algorithm can found in [9].

For the comparison purpose, we solved the dispatch problems, considered in the numerical example section, via the F-MSG method where the net water usage of the p-s unit is adjusted by controlling the pseudowater price [10]. In the FMSG method, the bus voltage magnitudes and phase angles, the off nominal tap settings, and the susceptances of the SVAR systems are taken as independent variables. Since all the constraints can be written in terms of those independent variables, the transmission line capacity constraints and bus voltage magnitude constraints are handled together in the same model easily. The load flow equations are imported into the model as equality constraints; therefore, the system loss is inserted into the solution process automatically. In the F-MSG algorithm, the upper bound for the cost function value is specified in advance, and the algorithm tries to find a solution where the cost function is less than or equal to the upper bound, while all constraints are satisfied (feasible solution). If it finds it, the upper bound is decreased by a certain amount; otherwise (infeasible solution) the upper bound is increased by a certain amount. The amount of decrease or increase on the upper bound for the next iteration depends on if any feasible or infeasible solution was obtained in the previous iterations. If the current solution is feasible and any infeasible solution has not been chosen yet, then the decrease amount on the upper bound for the next iteration remains the same. If the current solution is feasible and an infeasible solution has been chosen before, then the decrease amount on the upper bound for the next iteration is taken as the half of its previous value. If the current solution is infeasible and any feasible solution has not been chosen yet, then the increase amount on the upper bound for the next iteration remains the same. If the current solution is infeasible and a feasible solution has been chosen before, then the increase amount on the upper bound for the next iteration is taken as the half of its previous value. This process continues until absolute value of the change in the upper bound is less than a predefined tolerance value. Note that a specific initial increase/decrease amount for the cost function is selected at the beginning of the algorithm. To find the initial bus voltage magnitude and phase angles, a load flow calculation is performed with the selected initial unit generations at the beginning of the algorithm only. No more load flow calculation is performed in the subsequent stages of the proposed solution process. Since the F-MSG algorithm works with only the equality constraints, we convert inequality constraints into equality constraints by using a method which does not add any extra independent variable into the optimization model. As a summary, the dispatch problem of each subinterval is solved via the F-MSG method with the selected pseudowater price. After that, the net water usage by the p-s unit is calculated. If absolute value of the net water usage is less than a predetermined tolerance value, the solution procedure is terminated, and the solution is retained. If it is not, a new pseudowater price value is calculated via the bisection method, and a new iteration is started.

\section{Problem Formulation}

The optimization problem under consideration is solved in two steps as active power and reactive power optimization subproblems.

2.1. Active Power Optimization. The active power optimization problem, which is considered in this paper, can be given mathematically as follows ( $\mathrm{R}$ represents a fictitious monetary unit):

$$
\begin{aligned}
& \text { Minimize } F_{T}= w \sum_{j=1}^{j_{\max }}\left(\sum_{n \in \mathbf{N}_{s}} F_{n}\left(P_{n, j}\right)\right) t_{j} \\
&+(1-w) \xi \sum_{j=1}^{j_{\max }}\left(\sum_{n \in \mathbf{N}_{s}} E_{n}\left(P_{n, j}\right)\right) t_{j}, \\
& 0 \leq w \leq 1,(\mathrm{R})
\end{aligned}
$$

subject to hydraulic and electric constraints 


$$
\begin{gathered}
P_{\text {load }, j}+P_{\text {loss }, j}+P_{P H}-\sum_{n \in \mathbf{N}_{s}} P_{n, j}=0, \quad j \in \mathbf{J}_{\text {pump }}, \\
P_{\text {load }, j}+P_{\text {loss }, j}-P_{G H, j}-\sum_{n \in \mathbf{N}_{s}} P_{n, j}=0, \quad j \in \mathbf{J}_{\text {gen }}, \\
P_{n}^{\min } \leq P_{n, j} \leq P_{n}^{\max }, \quad n \in \mathbf{N}_{s}, \quad j=1, \ldots, j_{\max }, \\
\quad p_{l} \leq p_{l}^{\max }, \quad l \in \mathbf{N}_{\text {line }}, \\
P_{G H}^{\min } \leq P_{G H, j} \leq P_{G H}^{\max }, \quad j \in \mathbf{J}_{\text {gen }} \quad \text { or } \\
q_{G H}^{\min } \leq q_{G H}\left(P_{G H, j}\right) \leq q_{G H}^{\max }, \quad j \in \mathbf{J}_{\mathrm{gen}}, \\
P_{P H}^{\min } \leq P_{P H, j} \leq P_{P H}^{\max }, \quad j \in \mathbf{J}_{\text {pump }} \quad \text { or } \\
q_{P H}^{\min } \leq q_{P H}\left(P_{P H, j} \leq q_{P H}^{\max }, \quad j \in \mathbf{J}_{\text {pump }},\right. \\
V^{\min } \leq V_{j} \leq V^{\max }, \quad j=1, \ldots, j_{\text {max }}, \\
V_{j}=V_{j-1}+q_{P H}\left(P_{P H, j}\right) t_{j} \quad \text { if } j \in \mathbf{J}_{\text {pump}}, \\
V_{j}=V_{j-1}-q_{G H}\left(P_{G H, j}\right) t_{j} \quad \text { if } j \in \mathbf{J}_{\text {gen }}, \\
V_{0}=V_{j_{\max }}=V^{\text {start }}=V^{\text {end }} .
\end{gathered}
$$

Since the beginning and final water volume values of the upper reservoir of the p-s unit are taken as the same in the considered problem, the total net water amount used by the p-s unit must be equal to zero. That is to say, the total water amount, which is used for generation, must be equal to the total pumped water amount in an operation cycle.

$$
q_{\text {spent ТОт }}-q_{\text {pump ТОт }}=q_{\text {net spent }}=0 \text {, }
$$

where

$$
\begin{aligned}
q_{\text {spent TOT }} & =\sum_{j \in \mathbf{J}_{\text {gen }}} q_{G H}\left(P_{G H, j}\right) t_{j}, \\
q_{\text {pump TOT }} & =\sum_{j \in \mathbf{J}_{\text {pump }}} q_{P H}\left(P_{P H, j}\right) t_{j} .
\end{aligned}
$$

The meanings of the symbols used in the equations above are given in the list of symbol section.

2.2. Reactive Power Optimization. The reactive power optimization problem considered in this paper can be given mathematically as follows:

$$
\begin{aligned}
\text { Minimize } \quad F_{\mathrm{TOT}, \mathrm{sw}}= & w \sum_{j=1}^{j_{\max }} F_{\mathrm{sw}}\left(P_{\mathrm{sw}, j}\right) t_{j}+(1-w) \xi \\
& \times \sum_{j=1}^{j_{\max }} E_{\mathrm{sw}}\left(P_{\mathrm{sw}, j}\right) t_{j}
\end{aligned}
$$

subject to

$$
\begin{gathered}
Q_{\text {load }, j}+Q_{\text {loss }, j}+\sum_{m \in \mathbf{N}_{Q}} Q_{m, j}=0, \\
Q_{m}^{\min } \leq Q_{m, j} \leq Q_{m}^{\max }, \quad m \in \mathbf{N}_{Q}, \quad j=1, \ldots, j_{\mathrm{max}}, \\
P_{\mathrm{sw}}^{\min } \leq P_{\mathrm{sw}, j} \leq P_{\mathrm{sw}}^{\max }, \quad j=1, \ldots, j_{\mathrm{max}}, \\
U_{i}^{\min } \leq U_{i, j} \leq U_{i}^{\max }, \\
i \in \mathbf{N}_{\text {bus }}, \quad i \neq \mathrm{sw}, \quad i \notin \mathbf{N}_{v c}, \quad j=1, \ldots, j_{\max } .
\end{gathered}
$$

In the active and reactive power optimization, reactive power generation/consumption of the p-s unit in both modes is taken as zero for the comparison purpose of the results that are obtained from the systems with and without $\mathrm{p}$-s unit.

The meanings of the symbols used in the equations above are given in the list of symbol section.

\section{Application of Genetic Algorithm to the Optimization Problem}

The application of genetic algorithm to the optimization problem is achieved in two stages: application of genetic algorithm to the active and reactive power optimization subproblems.

3.1. Application of Genetic Algorithm to the Active Power Optimization. In application of genetic algorithm to the active power optimization problem, we used "Genetic Algorithm Tool Box" of MATLAB. All active power generation inequality constraints (except those which belong to the thermal unit connected to the swing bus) are handled by the mapping and coding mechanism of the genetic algorithm $[8,9]$. For an expensive thermal unit, although its minimum active power generation limit is $P_{n}^{\mathrm{min}}$, its active power inequality constraint is taken in the form of $0 \leq P_{n, j} \leq P_{n}^{\max }, j=1, \ldots, j_{\max }$. If the value of $P_{n, j}$, determined by the genetic algorithm, is less than $P_{n}^{\min }$, the unit is switched into synchronous compensator mode.

Active pumping/generation power limits of the p-s unit are taken as $0 \leq P_{p s, j} \leq\left(P_{P H}^{\max }+P_{G H}^{\max }\right), j=1, \ldots, j_{\max }$. If the value of $P_{p s, j}$, determined by the genetic algorithm, satisfies $0 \leq P_{p s, j} \leq P_{P H}^{\max }$, then $P_{p s, j}$ is directly taken as pumping (load) power $\left(P_{P H, j}=P_{p s, j}\right)$. If the value of $P_{p s, j}$, determined by the genetic algorithm, satisfies $P_{P H}^{\max }<P_{p s, j} \leq\left(P_{P H}^{\max }+P_{G H}^{\max }\right)$, then $P_{p s, j}$ is used in determination of the generation power as $P_{G H, j}=P_{p s, j}-P_{P H}^{\max }$.

The cost function, which will be minimized in the active power optimization, is taken as follows:

$$
f^{P}=F_{T}+p n f^{P}, \quad 0 \leq w \leq 1,
$$

where $F_{T}$ is given in (1) and $p n f^{P}$ represents the sum of various penalty functions. It is calculated as

$$
p n f^{P}=p n f_{\mathrm{sw}}^{\text {motor }}+p n f_{P_{\mathrm{sw}}}+p n f_{V}+p n f_{V^{\text {end }}}+p n f_{\text {line }} .
$$


$p n f_{\text {sw }}^{\text {motor }}$ in (16) is the penalty function, which takes comparably high values when the unit connected to the swing bus works as in motor mode. It is given as follows:

$$
p n f_{\mathrm{sw}}^{\mathrm{motor}}=K_{\mathrm{sw}}^{\text {motor }} \sum_{j \in\left\{P_{\mathrm{sw}, j}<0\right\}}\left(P_{\mathrm{sw}, j}\right)^{2} .
$$

$p n f_{P_{\mathrm{sw}}}$ in (16) represents the penalty function related to the active generation limits of the unit connected to the swing bus. It is calculated according to the following equation:

$$
\begin{gathered}
p n f_{P_{\mathrm{sw}}}=K_{P_{\mathrm{sw}}}\left(\sum_{j \in\left\{P_{\mathrm{sw}, j}<P_{\mathrm{sw}}^{\min }\right\}}\left(P_{\mathrm{sw}, j}-P_{\mathrm{sw}}^{\mathrm{min}}\right)^{2}\right. \\
\left.+\sum_{j \in\left\{P_{\mathrm{sw}, j}>P_{\mathrm{sw}}^{\max }\right\}}\left(P_{\mathrm{sw}, j}-P_{\mathrm{sw}}^{\mathrm{max}}\right)^{2}\right) .
\end{gathered}
$$

$p n f_{V}$ in (16) stands for the penalty function related to the reservoir storage constraints of the $\mathrm{p}$-s hydraulic unit. It is calculated as

$$
\begin{array}{r}
p n f_{V}=K_{V}\left(\sum_{j \in\left\{V_{j}<V^{\min }\right\}, j \neq j_{\max }}\left(V_{j}-V_{j}^{\min }\right)^{2}\right. \\
\left.+\sum_{j \in\left\{V_{j}>V^{\max }\right\}, j \neq j_{\max }}\left(V_{j}-V_{j}^{\max }\right)^{2}\right),
\end{array}
$$

where $V_{j}$ value is determined by (8). It should be remembered that the initial and final reservoir water volume values $\left(V_{0}\right.$ and $V_{j_{\max }}$ ) are taken as fixed and equal to each other.

$p n f_{V^{\text {end }}}$ in (16) denotes the penalty function, which is related to the water amount left in the upper reservoir of the p-s hydrounit at the end of the last subinterval. It is calculated as

$$
p n f_{V^{\text {end }}}=K_{V^{\text {end }}}\left(V_{j_{\max }}-V^{\text {end }}\right)^{2}
$$

$p n f_{\text {line }}$ in (16) represents the penalty function, which is concerned with the active power transmission capacities of the transmission lines, and it is calculated as

$$
p n f_{\text {line }}=\sum_{l \in \mathbf{N}_{\text {line }}} K_{\text {line }_{l}} \sum_{j \in\left\{p_{l, j}>p_{l}^{\max }\right\}}\left(p_{l, j}-p_{l}^{\max }\right)^{2} .
$$

$K_{\text {sw }}^{\text {motor }}, K_{P_{\text {sw }}}, K_{V}, K_{V^{\text {end }}}$, and $K_{\text {line }}, l \in \mathbf{N}_{\text {line }}$ in the above equations, are chosen positive penalty function constants.

In the active power optimization, reactive power generations of the units (except the thermal unit connected to the swing bus and the p-s unit) are taken as equal to arbitrarily selected feasible values. At the beginning, a random solution (which contains the active generations of all units (except the unit being connected to the reference bus) in all subintervals) is generated. After that, a Newton-Raphson load flow calculation is performed in each subinterval. Since the active generation of the unit connected to the swing bus and the active power line flows in each subinterval are known at this point, the fitness value of the solution is calculated. This process continues until an initial solution set is formed. Later, a new solution set is generated by using the fundamental operations of the genetic algorithm (selection, crossover, mutation, number of elite solution, etc.), and their fitness values are calculated $[8,9]$. The formation of a new solution set (generation) is repeated as many times as a selected number.

3.2. Application of Genetic Algorithm to the Reactive Power Optimization. In the application of genetic algorithm to the reactive power optimization problem, all reactive power generation inequality constraints (except those which belong to the thermal unit connected to the swing bus) are handled by the mapping and coding mechanism of the genetic algorithm $[8,9]$. The cost function, which will be minimized in the reactive power optimization, is taken as follows:

$$
f^{\mathrm{Q}}=F_{\mathrm{TOT}, \mathrm{sw}}+p n f^{\mathrm{Q}}, \quad 0 \leq w \leq 1,
$$

where $F_{\text {TOT,sw }}$ is given by (13) and $p n f^{\mathrm{Q}}$ stands for the sum of various penalty functions. It is calculated as follows:

$$
p n f^{\mathrm{Q}}=p n f_{P_{\mathrm{sw}}}+p n f_{\mathrm{Q}_{\mathrm{sw}}}+p n f_{U} .
$$

pnf $f_{P_{\mathrm{sw}}}$ in (23) has been already given in (18). Equation (24) gives the penalty function, which is related to the reactive power generation limits of the thermal unit connected to the swing bus as follows:

$$
\begin{array}{r}
p n f_{P_{\mathrm{sw}}}=K_{\mathrm{Q}_{\mathrm{sw}}}\left(\sum_{j \in\left\{\mathrm{Q}_{\mathrm{sw}, j}<\mathrm{Q}_{\mathrm{sw}}^{\min }\right\}}\left(Q_{\mathrm{sw}, j}-Q_{\mathrm{sw}}^{\min }\right)^{2}\right. \\
\left.+\sum_{j \in\left\{\mathrm{Q}_{\mathrm{sw}, j}>\mathrm{Q}_{\mathrm{sw}}^{\max }\right\}}\left(Q_{\mathrm{sw}, j}-Q_{\mathrm{sw}}^{\mathrm{max}}\right)^{2}\right) .
\end{array}
$$

Penalty function concerning with the voltage magnitude limits of the buses (except swing and voltage controlled buses) is given as follows:

$$
\begin{array}{r}
p n f_{U}=\sum_{\substack{i \in \mathbf{N}_{\text {bus }} \\
i \neq s w, i \neq \mathbf{N}_{v c}}} K_{U_{i}}\left[\sum_{j \in\left\{U_{i, j}<U_{i}^{\min }\right\}}\left(U_{i}^{\min }-U_{i, j}\right)^{2}\right. \\
\left.+\sum_{j \in\left\{U_{i, j}>U_{i}^{\max }\right\}}\left(U_{i, j}-U_{i}^{\max }\right)^{2}\right] .
\end{array}
$$

$K_{\mathrm{Q}_{\mathrm{sw}}}$ and $K_{U_{i}}, i \in \mathbf{N}_{\text {bus }}, i \neq \mathrm{sw}$, and $i \notin \mathbf{N}_{v c}$ in (24) and (25) are selected positive penalty function constants.

During the reactive power optimization calculations, active generation powers of the thermal units (except the one connected to the swing bus) and the active generation and pumping powers of the $\mathrm{p}$-s unit are taken as equal to those values determined in the active power optimization 
procedure. They are not changed. Reactive power generations of the thermal units (except the one connected to the swing bus) and reactive power sources are determined by the genetic algorithm. Since changing the reactive power generations affects the active and reactive transmission loss and voltage magnitudes of buses and since those losses are supplied by the unit connected to the swing bus, the cost function to be minimized is selected as the one shown in (22). The application of the genetic algorithm to the reactive power optimization problem resembles the one that is explained in Section 3.1.

\section{Numerical Example}

In order to demonstrate our proposed solution technique, an example power system, whose one-line diagram shown in Figure 7, is selected. Per unit line data, unit types, system base values, and load locations are also shown in the same figure. Thermal units are connected to buses $1,4,7,9$, and 11 . Their fuel cost rate and emission rate curves are taken as follows:

$$
\begin{aligned}
F_{n}\left(P_{G n}\right)=a_{n} & +b_{n} P_{G n}+c_{n} P_{G n}^{2}(\mathrm{R} / \mathrm{h}), \quad n=1,4,7,9,11, \\
E_{n}\left(P_{G n}\right)= & 10^{-2}\left(\alpha_{n}+\beta_{n} P_{G n}+\gamma_{n} P_{G n}^{2}\right) \\
& +\zeta_{n} e^{\left(\lambda_{n} P_{G n}\right)}(\mathrm{kg} / \mathrm{h}), \quad n=1,4,7,9,11 .
\end{aligned}
$$

The cost rate and emission rate curve coefficients of the units and their active and reactive power generation limits are given in Table 5. The active generation power in (26) are in pu. It is seen from the table that thermal units 9 and 11 are expensive units compared to the other thermal units in terms of both their fuel and emission cost rate values. Therefore, it is assumed that they can be used as synchronous compensators if their active generation powers determined by the genetic algorithm become less than their respective lower active generation limits.

The p-s hydraulic unit is connected to bus 6 . The p-s hydraulic unit scheduling problem considered in this paper is solved for three different p-s unit cycle efficiencies; namely, $\eta_{c}=0.60,0.67,0.75$. The discharge and pumping rate curves of the p-s unit are taken as in the following forms:

$$
\begin{aligned}
& q_{G H}\left(P_{G H}\right) \\
& =\left\{\begin{array}{lll}
200+2.0 P_{G H} & (\text { acre-ft }) & \text { if } 0<P_{G H} \leq 130 \mathrm{MW}, \\
0 & (\text { acre-ft }) & \text { if } P_{G H}=0 \mathrm{MW},
\end{array}\right. \\
& q_{P H}\left(P_{P H}\right) \\
& =\left\{\begin{array}{lll}
200+\mu P_{P H} & (\text { acre-ft }) & \text { if } 0<P_{P H} \leq 130 \mathrm{MW}, \\
0 & (\text { acre-ft }) & \text { if } P_{P H}=0 \mathrm{MW},
\end{array}\right.
\end{aligned}
$$

where $\mu$ is taken as equal to $6 / 5,4 / 3$, and $3 / 2$ for $60 \%, 67 \%$, and $75 \%$ cycle efficiencies, respectively. For the comparison purpose of the results obtained from the power systems with and without the p-s unit, the reactive power in pumping and
TABLE 1: Some chosen methods and parameters of the genetic algorithm that is used in the paper.

\begin{tabular}{lc}
\hline Parameter & Value \\
\hline$K_{\mathrm{sw}}^{\text {motor }}$ & $1 \times 10^{6}$ \\
$K_{P_{\mathrm{sw}}}$ & 0.8 \\
$K_{\mathrm{Q}_{\mathrm{sw}}}$ & 1.2 \\
$K_{V}$ & 0.6 \\
$K_{V^{\text {end }}}$ & 0.53 \\
$K_{\text {line }}, \forall l \in \mathbf{N}_{\text {line }}$ & 1000 \\
$K_{U_{i}}, \forall i \in \mathbf{N}_{\text {bus }}$ & $1 \times 10^{8}$ \\
$U_{i}^{\min }(\mathrm{pu}), \forall i \in \mathbf{N}_{\text {bus }}, i \neq \mathrm{sw}$ & 0.9 \\
$U_{i}^{\text {max }}(\mathrm{pu}), \forall i \in \mathbf{N}_{\text {bus }}, i \neq \mathrm{sw}$ & 1.07 \\
$p_{l}^{\text {max }}, \forall l \in \mathbf{N}_{\text {line }}(\mathrm{MW})$ & 81 \\
Population & 150 \\
Number of generation & 150 \\
Number of elite solution & 1 \\
Mutation method and probability & Uniform, 0.0001 \\
Crossover method and probability & Scattered, 0.9999 \\
Selection method & Stochastic uniform \\
\hline
\end{tabular}

generation mode of the p-s unit is taken as 0 MVAr. Data related to the upper reservoir of the p-s unit is taken as $V^{\min }=5000$ acre-ft, $V^{\max }=15000$ acre- $\mathrm{ft}$, and $V_{0}=V_{6}=$ 10000 acre-ft.

Twenty-four-hour operation period having six equal subintervals was considered: $t_{j}=4 \mathrm{~h}, j=1,2, \ldots, 6$. The load schedule of the example power system is given in Table 6 . We see from the table that off-peak load levels occur in subintervals 1, 5, and 6 and peak-load levels occur in subintervals 2,3 , and 4 .

The problem is solved for the different weight coefficients $(w=0,0.1, \ldots, 0.9,1.0)$ under the condition that there is no $\mathrm{p}$-s unit in the power system first. It is observed in those Pareto optimal solutions that all penalty function values in (16) and (23) take zero value. Also it is seen that thermal units 9 and 11 (expensive units) work in generator mode only in subinterval 3 (where the peak loads occur) and in synchronous compensator mode in the other subintervals. Later on, the same Pareto optimal solutions are recalculated for the case where there is p-s unit with specific cycle efficiency in the example power system. The last solution is done for three different cycle efficiencies of the $\mathrm{p}$-s unit $\left(\eta_{c}=0.6,0.67,0.75\right)$. All penalty function values of $(16)$ and (23) (except the one belongs to $V_{j_{\max }}=V_{6}$ ) in all solutions are observed as zero. The maximum error related to $V_{6}$ among all solutions is obtained as equal to $0.0336 \%$ of $10000 \mathrm{ccf}$.

Some chosen methods and parameters of the genetic algorithm that is used in this paper are given in Table 1 .

Trade-off curves, for the case where there is no p-s hydraulic unit in the power system, are given in Figure 1. In the figure, trade-off curves that are found just after the active power optimization and the reactive power optimization are shown. Extra decrease obtained in both total fuel and emission costs due to performed reactive power optimization is clearly seen from the figure. 
TABLE 2: Various results obtained after the active power and reactive power optimizations for the case where there is no p-s unit in the power system and $w=0.5$.

\begin{tabular}{|c|c|c|c|c|c|c|c|c|c|c|c|c|}
\hline \multirow[b]{3}{*}{$j$} & \multicolumn{12}{|c|}{$w=0.5$} \\
\hline & \multicolumn{6}{|c|}{ Active power optimization solution point values } & \multicolumn{6}{|c|}{ Reactive power optimization solution point values } \\
\hline & 1 & 2 & 3 & 4 & 5 & 6 & 1 & 2 & 3 & 4 & 5 & 6 \\
\hline$P_{\text {load }, j}$ & 200 & 600 & 700 & 600 & 300 & 200 & 200 & 600 & 700 & 600 & 300 & 200 \\
\hline$P_{G 1, j}$ & 104.43 & 290.44 & 320.39 & 289.15 & 149.58 & 103.76 & 104.53 & 287.28 & 315.77 & 285.92 & 148.26 & 103.84 \\
\hline$Q_{G 1, j}$ & 49.77 & 176.50 & 195.99 & 179.33 & 108.25 & 49.70 & -5.71 & 90.04 & 97.91 & 86.69 & 11.65 & -6.07 \\
\hline$U_{1, j}$ & 1.050 & 1.050 & 1.050 & 1.050 & 1.050 & 1.050 & 1.050 & 1.050 & 1.050 & 1.050 & 1.050 & 1.050 \\
\hline$U_{2, j}$ & 1.015 & 0.941 & 0.923 & 0.942 & 0.981 & 1.015 & 1.037 & 0.977 & 0.963 & 0.978 & 1.020 & 1.037 \\
\hline$U_{3, j}$ & 0.995 & 0.910 & 0.901 & 0.910 & 0.963 & 0.995 & 1.019 & 0.951 & 0.945 & 0.952 & 1.007 & 1.020 \\
\hline$P_{G 4, j}$ & 48.66 & 178.06 & 179.80 & 179.31 & 79.39 & 47.21 & 48.66 & 178.06 & 179.80 & 179.31 & 79.39 & 47.21 \\
\hline$Q_{G 4, j}$ & 20.00 & 90.00 & 110.00 & 90.00 & 30.00 & 20.00 & 28.68 & 112.37 & 126.05 & 108.48 & 51.44 & 32.98 \\
\hline$U_{4, j}$ & 1.031 & 1.017 & 1.015 & 1.017 & 1.006 & 1.031 & 1.063 & 1.070 & 1.070 & 1.070 & 1.066 & 1.065 \\
\hline$U_{5, j}$ & 1.011 & 0.918 & 0.921 & 0.918 & 0.995 & 1.011 & 1.027 & 0.944 & 0.951 & 0.945 & 1.023 & 1.027 \\
\hline$P_{G H, j}$ & 0.00 & 0.00 & 0.00 & 0.00 & 0.00 & 0.00 & 0.00 & 0.00 & 0.00 & 0.00 & 0.00 & 0.00 \\
\hline$P_{P H, j}$ & 0.00 & 0.00 & 0.00 & 0.00 & 0.00 & 0.00 & 0.00 & 0.00 & 0.00 & 0.00 & 0.00 & 0.00 \\
\hline$Q_{p s, j}$ & 0.00 & 0.00 & 0.00 & 0.00 & 0.00 & 0.00 & 0.00 & 0.00 & 0.00 & 0.00 & 0.00 & 0.00 \\
\hline$U_{6, j}$ & 1.030 & 0.980 & 0.965 & 0.980 & 1.004 & 1.030 & 1.047 & 1.007 & 0.998 & 1.008 & 1.035 & 1.047 \\
\hline$P_{G 7, j}$ & 50.72 & 169.77 & 169.96 & 169.75 & 80.89 & 52.62 & 50.72 & 169.77 & 169.96 & 169.75 & 80.89 & 52.62 \\
\hline$Q_{G 7, j}$ & 20.00 & 90.00 & 110.00 & 90.00 & 30.00 & 20.00 & 37.79 & 112.80 & 135.91 & 115.85 & 58.03 & 37.36 \\
\hline$U_{7, j}$ & 1.024 & 0.974 & 0.962 & 0.974 & 0.990 & 1.025 & 1.054 & 1.019 & 1.015 & 1.022 & 1.042 & 1.054 \\
\hline$U_{8, j}$ & 0.997 & 0.918 & 0.904 & 0.918 & 0.956 & 0.997 & 1.038 & 0.981 & 0.977 & 0.984 & 1.026 & 1.037 \\
\hline$P_{G, j}$ & 0.00 & 0.00 & 43.28 & 0.00 & 0.00 & 0.00 & 0.00 & 0.00 & 43.28 & 0.00 & 0.00 & 0.00 \\
\hline$Q_{G 9, j}$ & 20.00 & 90.00 & 110.00 & 90.00 & 30.00 & 20.00 & 32.59 & 103.70 & 120.15 & 102.76 & 49.37 & 30.76 \\
\hline$U_{9, j}$ & 1.014 & 0.972 & 0.987 & 0.972 & 0.972 & 1.014 & 1.060 & 1.038 & 1.059 & 1.039 & 1.051 & 1.058 \\
\hline$U_{10, j}$ & 1.014 & 0.924 & 0.871 & 0.924 & 0.952 & 1.014 & 1.046 & 0.973 & 0.934 & 0.976 & 1.007 & 1.045 \\
\hline$P_{G 11, j}$ & 0.00 & 0.00 & 31.81 & 0.00 & 0.00 & 0.00 & 0.00 & 0.00 & 31.81 & 0.00 & 0.00 & 0.00 \\
\hline$Q_{G 11, j}$ & 20.00 & 90.00 & 110.00 & 90.00 & 30.00 & 20.00 & 34.47 & 105.21 & 133.81 & 109.80 & 50.22 & 32.68 \\
\hline$U_{11, j}$ & 1.021 & 0.968 & 0.963 & 0.968 & 0.970 & 1.021 & 1.065 & 1.031 & 1.042 & 1.036 & 1.044 & 1.063 \\
\hline$U_{12, j}$ & 0.997 & 0.866 & 0.859 & 0.867 & 0.921 & 0.997 & 1.042 & 0.938 & 0.948 & 0.942 & 0.999 & 1.041 \\
\hline Fuel cost ( $R)$ & 915.17 & 3533.50 & 5130.50 & 3532.90 & 1422.10 & 915.55 & 915.57 & 3509.00 & 5091.90 & 3507.80 & 1415.50 & 915.86 \\
\hline Emission cost (R) & 208.99 & 651.96 & 1435.10 & 652.60 & 176.39 & 208.94 & 208.94 & 644.15 & 1421.40 & 644.64 & 176.13 & 208.90 \\
\hline Total fuel cost (R) & \multicolumn{6}{|c|}{15450.00} & \multicolumn{6}{|c|}{15356.00} \\
\hline Total emission cost (R) & \multicolumn{6}{|c|}{3334.00} & \multicolumn{6}{|c|}{3304.20} \\
\hline Total cost $(\mathrm{R})$ & \multicolumn{6}{|c|}{18784.00} & \multicolumn{6}{|c|}{18660.20} \\
\hline
\end{tabular}

Various results obtained after active power and reactive power optimizations, for the case where there is no $\mathrm{p}$-s unit in the power system and $w=0.5$, are given in Table 2 just as an example. The effect of the reactive power optimization on the improvement of the bus voltage magnitudes can be seen from the table. The chosen reactive power generations in all subintervals during the active power optimization are also given in Table 2 . We see from the table that expensive units (units 9 and 11) generate active power only in subinterval 3 , where the peak load occurs and work as synchronous compensator in the other subintervals.

The change in the total cost decrease percentage (with respect to the case where there is no $\mathrm{p}$-s unit in the power system) versus $w$ is shown in Figure 2, where the p-s unit cycle efficiency is taken as parameter. It is seen from the figure that the higher the cycle efficiency the p-s unit has, the higher the decrease percentage in the total cost we get.

As an example, the solution point pumping and generation powers of the $\mathrm{p}$-s unit together with the total active load values during all subintervals for $w=0.5$ are given in Figure 3 where the p-s unit cycle efficiency is taken as parameter. The p-s unit works in pumping mode in low load valued intervals and in generation mode in high load valued intervals for all cycle efficiencies. We see from Table 3 that although the p-s unit with $75 \%$ cycle efficiency consumes less amount of total pumping energy compared to the units with the lower cycle efficiencies, it produces higher total generation energy. Because of this, it gives higher decrease percentage on the total cost when it is employed in the power system. 
TABLE 3: Generation and pumping energy, final water amount in the upper reservoir versus p-s cycle efficiency for $w=0.5$.

\begin{tabular}{lccc}
\hline Cycle efficiency $(\%)$ & Generation energy $(\mathrm{MWh})$ & Pumping energy $(\mathrm{MWh})$ & $V_{6}(\mathrm{acre}-\mathrm{ft})$ \\
\hline 60 & 795.08 & 1324.16 & 9998.80 \\
67 & 803.84 & 1208.28 & 10003.36 \\
75 & 842.72 & 1123.38 & 9999.63 \\
\hline
\end{tabular}

TABLE 4: Various results obtained after the active power and reactive power optimizations for the case where there is $\mathrm{p}$-s unit with $75 \%$ cycle efficiency in the power system and $w=0.5$.

\begin{tabular}{|c|c|c|c|c|c|c|c|c|c|c|c|c|}
\hline \multirow[b]{3}{*}{$j$} & \multicolumn{12}{|c|}{$w=0.5$} \\
\hline & \multicolumn{6}{|c|}{ Active power optimization solution point values } & \multicolumn{6}{|c|}{ Reactive power optimization solution point values } \\
\hline & 1 & 2 & 3 & 4 & 5 & 6 & 1 & 2 & 3 & 4 & 5 & 6 \\
\hline$P_{\text {load }, j}$ & 200 & 600 & 700 & 600 & 300 & 200 & 200 & 600 & 700 & 600 & 300 & 200 \\
\hline$P_{G 1, j}$ & 151.02 & 273.51 & 277.92 & 256.79 & 167.78 & 129.67 & 150.72 & 270.45 & 269.61 & 253.92 & 166.27 & 129.64 \\
\hline$Q_{G 1, j}$ & 64.60 & 172.61 & 234.25 & 170.16 & 113.00 & 55.33 & 13.39 & 85.93 & 115.50 & 81.54 & 22.18 & 4.40 \\
\hline$U_{1, j}$ & 1.050 & 1.050 & 1.050 & 1.050 & 1.050 & 1.050 & 1.050 & 1.050 & 1.050 & 1.050 & 1.050 & 1.050 \\
\hline$U_{2, j}$ & 1.016 & 0.943 & 0.904 & 0.943 & 0.983 & 1.017 & 1.036 & 0.978 & 0.953 & 0.979 & 1.020 & 1.037 \\
\hline$U_{3, j}$ & 0.996 & 0.911 & 0.875 & 0.911 & 0.965 & 0.997 & 1.019 & 0.952 & 0.931 & 0.954 & 1.007 & 1.020 \\
\hline$P_{G 4, j}$ & 108.89 & 169.10 & 179.44 & 155.21 & 87.23 & 85.47 & 108.89 & 169.10 & 179.44 & 155.21 & 87.23 & 85.47 \\
\hline$Q_{G 4, j}$ & 20.00 & 90.00 & 110.00 & 90.00 & 30.00 & 20.00 & 29.84 & 110.89 & 130.55 & 109.97 & 50.32 & 28.23 \\
\hline$U_{4, j}$ & 1.038 & 1.018 & 0.991 & 1.017 & 1.009 & 1.036 & 1.068 & 1.070 & 1.059 & 1.070 & 1.065 & 1.066 \\
\hline$U_{5, j}$ & 0.999 & 0.921 & 0.917 & 0.924 & 0.992 & 1.005 & 1.014 & 0.947 & 0.953 & 0.951 & 1.017 & 1.020 \\
\hline$P_{G H, j}$ & - & 24.44 & 129.83 & 56.41 & - & - & - & 24.44 & 129.83 & 56.41 & - & - \\
\hline$P_{P H, j}$ & 127.86 & - & - & - & 76.993 & 75.992 & 127.86 & - & - & - & 76.99 & 75.99 \\
\hline$Q_{p s, j}$ & 0.00 & 0.00 & 0.00 & 0.00 & 0.00 & 0.00 & 0.00 & 0.00 & 0.00 & 0.00 & 0.00 & 0.00 \\
\hline$U_{6, j}$ & 1.000 & 0.986 & 0.975 & 0.993 & 0.990 & 1.014 & 1.016 & 1.013 & 1.013 & 1.021 & 1.019 & 1.030 \\
\hline$P_{G 7, j}$ & 76.67 & 169.90 & 169.97 & 167.71 & 133.43 & 66.53 & 76.67 & 169.90 & 169.97 & 167.71 & 133.43 & 66.53 \\
\hline$Q_{G 7, j}$ & 20.00 & 90.00 & 110.00 & 90.00 & 30.00 & 20.00 & 34.00 & 116.63 & 136.09 & 113.63 & 53.00 & 34.09 \\
\hline$U_{7, j}$ & 1.019 & 0.977 & 0.944 & 0.979 & 0.992 & 1.023 & 1.046 & 1.023 & 1.006 & 1.025 & 1.040 & 1.050 \\
\hline$U_{8, j}$ & 0.994 & 0.920 & 0.861 & 0.922 & .957 & 00.997 & 1.032 & 0.983 & 0.953 & 0.988 & 1.024 & 1.035 \\
\hline$P_{G 9, j}$ & 0.00 & 0.00 & 0.00 & 0.00 & 0.00 & 0.00 & 0.00 & 0.00 & 0.00 & 0.00 & 0.00 & 0.00 \\
\hline$Q_{G 9, j}$ & 20.00 & 90.00 & 110.00 & 90.00 & 30.00 & 20.00 & 30.84 & 103.27 & 128.63 & 107.24 & 50.53 & 31.51 \\
\hline$U_{9, j}$ & 1.011 & 0.974 & 0.939 & 0.975 & 0.973 & 1.014 & 1.054 & 1.039 & 1.032 & 1.046 & 1.050 & 1.057 \\
\hline$U_{10, j}$ & 1.001 & 0.928 & 0.847 & 0.931 & 0.948 & 1.008 & 1.031 & 0.977 & 0.921 & 0.982 & 1.001 & 1.038 \\
\hline$P_{G 11, j}$ & 0.00 & 0.00 & 0.00 & 0.00 & 0.00 & 0.00 & 0.00 & 0.00 & 0.00 & 0.00 & 0.00 & 0.00 \\
\hline$Q_{G 11, j}$ & 20.00 & 90.00 & 110.00 & 90.00 & 30.00 & 20.00 & 33.67 & 103.77 & 134.72 & 106.12 & 49.08 & 35.01 \\
\hline$U_{11, j}$ & 1.014 & 0.971 & 0.924 & 0.973 & 0.970 & 1.018 & 1.055 & 1.033 & 1.017 & 1.038 & 1.040 & 1.060 \\
\hline$U_{12, j}$ & 0.993 & 0.869 & 0.810 & 0.871 & 0.921 & 0.996 & 1.034 & 0.939 & 0.914 & 0.946 & 0.997 & 1.039 \\
\hline$V_{j}$ & 11567.00 & 10572.00 & 8733.00 & 7481.60 & 8743.60 & 9999.50 & 11567.00 & 10572.00 & 8733.00 & 7481.60 & 8743.60 & 9999.50 \\
\hline Fuel cost $(\mathrm{R})$ & 1573.90 & 3337.40 & 3449.40 & 3097.60 & 1872.40 & 1285.10 & 1572.50 & 3314.70 & 3387.70 & 3077.30 & 1864.30 & 1285.00 \\
\hline Emission cost (R) & 191.12 & 585.72 & 626.76 & 508.31 & 231.16 & 178.28 & 191.05 & 578.99 & 608.33 & 502.79 & 230.41 & 178.28 \\
\hline Total fuel cost (R) & & & 14616 & 6.00 & & & & & 14501 & 1.00 & & \\
\hline Total emission cost ( $\mathrm{R})$ & & & 2321 & 1.40 & & & & & 2289 & 9.70 & & \\
\hline Total cost $(\mathrm{R})$ & & & 16937 & 7.40 & & & & & 16790 & 0.70 & & \\
\hline
\end{tabular}

The change of stored water amount in the upper reservoir of the p-s unit versus time for $w=0.5$ is shown in Figure 4 where $\mathrm{p}$-s unit cycle efficiency is taken as parameter. From the figure, it is seen that the water amount used by the pumped storage unit in the power system under consideration does not strongly depend on the p-s unit's cycle efficiency. The stored water volume values in the upper reservoir remain inside its upper and lower storage limits $\left(V^{\min }=5000\right.$ acre-ft, $V^{\max }=15000$ acre-ft $)$. The final stored water amounts in the upper reservoir for all cycle efficiencies are very close to $V^{\text {end }}=10000$ acre-ft (see the last column in Table 3 ). Various results, which are obtained after the active and reactive power optimizations for $w=0.5$ and $75 \%$ p-s unit cycle efficiency, are given in Table 4 . It is 
TABLE 5: Cost rate and emission rate curve coefficients of the thermal units and their active and reactive power generation limits.

\begin{tabular}{|c|c|c|c|c|c|c|}
\hline \multirow{2}{*}{ Curve } & \multirow{2}{*}{ Constants } & \multicolumn{5}{|c|}{ Thermal unit number, $(n)$} \\
\hline & & 1 & 4 & 7 & 9 & 11 \\
\hline \multirow{3}{*}{ Fuel cost rate } & $a_{n}$ & 10 & 20 & 20 & 70 & 70 \\
\hline & $b_{n}$ & 50 & 85 & 85 & 215 & 205 \\
\hline & $c_{n}$ & 25 & 30 & 30 & 80 & 213 \\
\hline \multirow{6}{*}{ Emission rate } & $\alpha_{n}$ & 4.426 & 4.258 & 4.258 & 7.743 & 8.531 \\
\hline & $\beta_{n}$ & -5.550 & -6.094 & -6.094 & -2.147 & -2.355 \\
\hline & $\gamma_{n}$ & 2.030 & 3.886 & 3.886 & 8.438 & 8.651 \\
\hline & $\zeta_{n}$ & $2.0 \times 10^{-6}$ & $1.0 \times 10^{-6}$ & $1.0 \times 10^{-6}$ & $5.0 \times 10^{-5}$ & $5.0 \times 10^{-5}$ \\
\hline & $\lambda_{n}$ & 1.5 & 2.35 & 2.35 & 8.833 & 8.967 \\
\hline & $\xi$ & & & 1000 & & \\
\hline \multirow{4}{*}{ Generation limits } & $P_{n}^{\min }(\mathrm{MW})$ & 50.0 & 45.0 & 40.0 & 5.0 & 5.0 \\
\hline & $P_{n}^{\max }(\mathrm{MW})$ & 320.0 & 180.0 & 170.0 & 100.0 & 100.0 \\
\hline & $Q_{n}^{\min }(\mathrm{MW})$ & -50 & -20 & -20 & -20 & -20 \\
\hline & $Q_{n}^{\max }(\mathrm{MW})$ & 150 & 200 & 200 & 200 & 200 \\
\hline
\end{tabular}

TABLE 6: Per unit load schedule for the example power system.

\begin{tabular}{|c|c|c|c|c|c|c|c|}
\hline \multirow{2}{*}{ Bus no. } & \multirow{2}{*}{ Load type } & \multicolumn{6}{|c|}{ Time subinterval number, $(j)$} \\
\hline & & 1 & 2 & 3 & 4 & 5 & 6 \\
\hline \multirow{2}{*}{2} & Active & 0.20 & 0.80 & 1.00 & 0.80 & 0.40 & 0.20 \\
\hline & Reactive & 0.15 & 0.60 & 0.75 & 0.60 & 0.30 & 0.15 \\
\hline \multirow{2}{*}{3} & Active & 0.50 & 1.10 & 1.20 & 1.10 & 0.60 & 0.50 \\
\hline & Reactive & 0.40 & 0.85 & 0.90 & 0.85 & 0.45 & 0.40 \\
\hline \multirow{2}{*}{5} & Active & 0.30 & 0.90 & 0.80 & 0.90 & 0.25 & 0.30 \\
\hline & Reactive & 0.24 & 0.70 & 0.60 & 0.70 & 0.20 & 0.24 \\
\hline \multirow{2}{*}{7} & Active & 0.25 & 1.00 & 1.10 & 1.00 & 0.50 & 0.25 \\
\hline & Reactive & 0.20 & 0.75 & 0.85 & 0.75 & 0.40 & 0.20 \\
\hline \multirow{2}{*}{8} & Active & 0.40 & 0.70 & 0.90 & 0.70 & 0.30 & 0.40 \\
\hline & Reactive & 0.30 & 0.52 & 0.70 & 0.52 & 0.24 & 0.30 \\
\hline \multirow{2}{*}{10} & Active & 0.15 & 0.60 & 1.05 & 0.60 & 0.45 & 0.15 \\
\hline & Reactive & 0.12 & 0.45 & 0.80 & 0.45 & 0.35 & 0.12 \\
\hline \multirow{2}{*}{12} & Active & 0.20 & 0.90 & 0.95 & 0.90 & 0.50 & 0.20 \\
\hline & Reactive & 0.15 & 0.70 & 0.75 & 0.70 & 0.40 & 0.15 \\
\hline \multirow{2}{*}{\multicolumn{2}{|c|}{$\begin{array}{l}P_{\text {load }, j}(\mathrm{pu}) \\
Q_{\text {load }, j}(\mathrm{pu})\end{array}$}} & 2.00 & 6.00 & 7.00 & 6.00 & 3.00 & 2.00 \\
\hline & & 1.56 & 4.57 & 5.35 & 4.57 & 2.34 & 1.56 \\
\hline
\end{tabular}

seen from the table that the expensive units never generate any active power but work as synchronous compensator in all subintervals. The generation and pumping active powers of the p-s unit are also given in the table. At the end of the active power optimization, the total cost (which is made up the total fuel cost plus the total emission cost) is decreased from 18660.20 (no p-s case with $w=0.5$ after the reactive power optimization) to $16937.4 \mathrm{R}$ by employing the $\mathrm{p}$-s unit with $75 \%$ cycle efficiency in the power system. Optimal reactive power generations that are determined just after the reactive power optimization are also shown in Table 4 . The total cost is decreased further from $16937.4 \mathrm{R}$ to $16790.70 \mathrm{R}$ at the end of the reactive power optimization. Consequently, $1869.5 \mathrm{R}$
(10.01\% of the total cost in no p-s case with $w=0.5$ after the reactive power optimization) is saved from $24 \mathrm{~h}$ operation of the p-s unit with $75 \%$ cycle efficiency in the power system.

4.1. Application of the F-MSG Method to the Same Dispatch Problems. The dispatch problems considered in Section 4 is resolved by means of the F-MSG method under the same constraints considered in Section 4. Since the active and reactive power optimizations are accomplished in the same model in the F-MSG method, there is no need to solve the dispatch problem in two stages. The trade-off curve obtained via the F-MSG method is shown in Figure 1 together with 


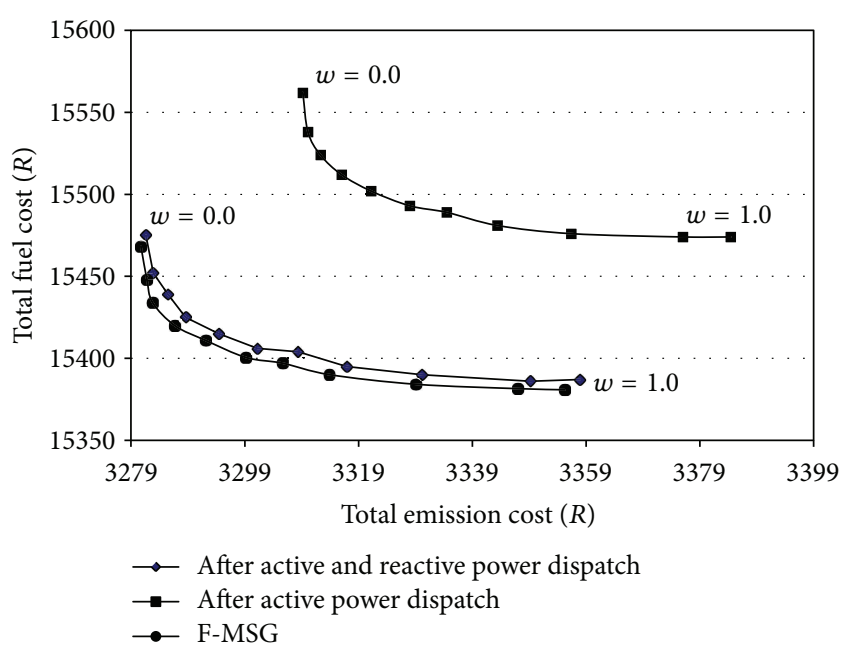

FIGURE 1: Trade-off curves for the case where no p-s unit exists in the example power system.

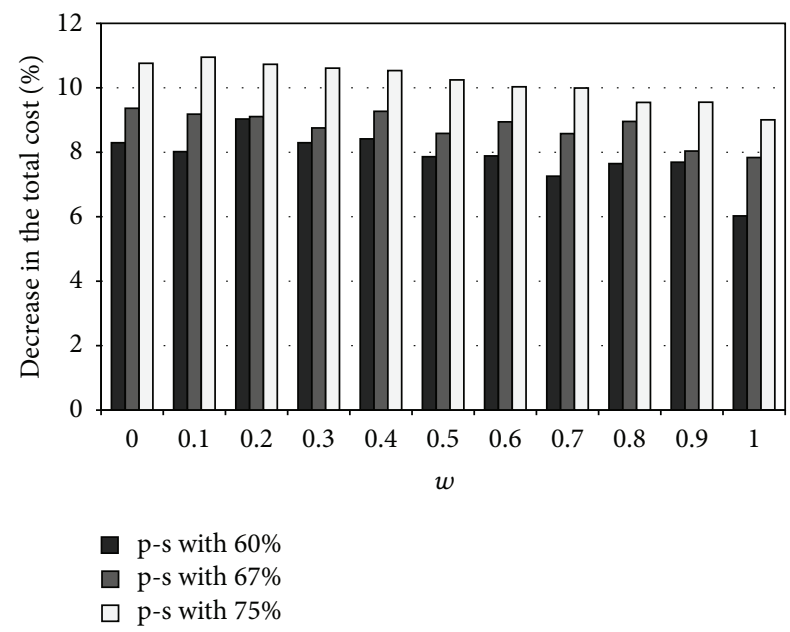

FIGURE 2: Change in the total cost decrease percentage with respect to $w$ for different $\mathrm{p}$-s unit cycle efficiencies.

the one found by means of the genetic algorithm. We see from the figure that this trade-off curve is very close to the one obtained after the active and reactive power optimizations by means of the method proposed in this paper. Once the dispatch problem of the example power system with p-s unit is resolved with the F-MSG, the obtained change in the total cost decrease percentage (with respect to the case where there is no p-s unit in the power system) versus $w$ is shown in Figure 5, where the p-s unit cycle efficiency is taken as parameter. From the comparison of Figure 5 with Figure 2, it is seen that the F-MSG method gives similar but a little bit higher decrease percentages. This is because the F-MSG method handles active and reactive power optimizations in the same model, whereas the method given in this paper considers either the active or reactive power optimizations at a time. The decrease percentages on the total cost can be

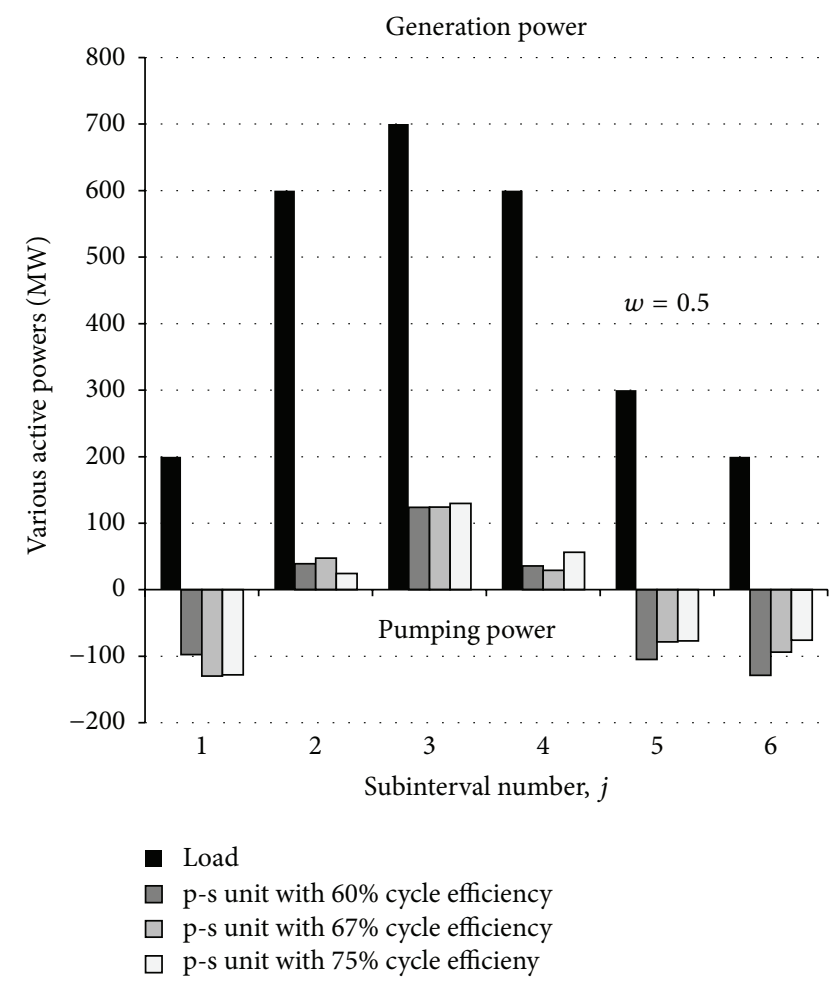

FIGURE 3: Active load and solution point pumping and generation powers of the p-s unit during six subintervals.



FIGURE 4: Change of stored water amounts in the upper reservoir with respect to time for $w=0.5$.

increased by performing the active and then reactive power optimizations in an iterative manner with the cost of longer solution times in the proposed solution method.

\section{Results and Conclusion}

Security constrained environmental p-s hydraulic unit scheduling problem is solved via genetic algorithm. The solution 


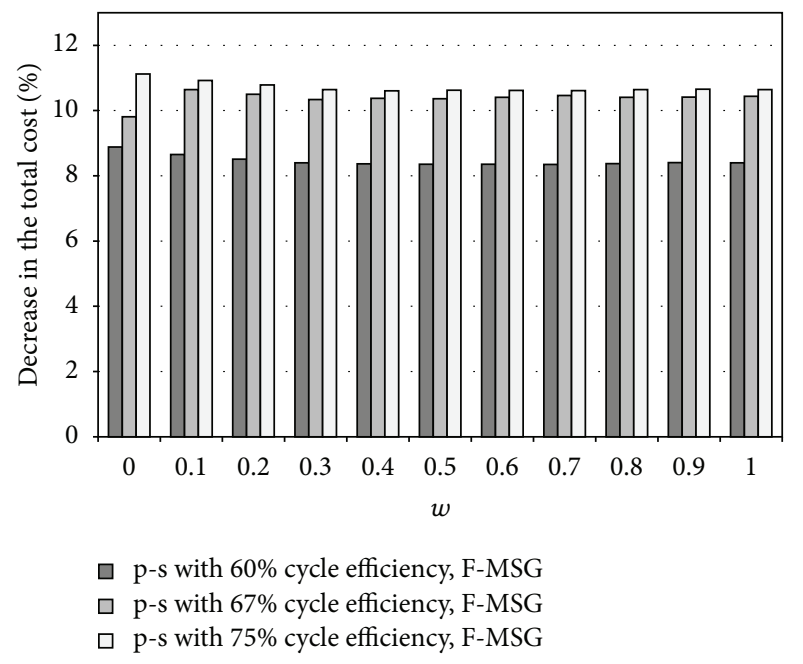

FIGURE 5: Change in the total cost decrease percentage with respect to $w$ for different $\mathrm{p}$-s unit cycle efficiencies, once the dispatch problem of the example power system with p-s unit is solved via the F-MSG method.

process is divided into two parts as active power and reactive power optimizations. Basically in the active power optimization, for arbitrarily selected reactive power generations, active power generations of the thermal units and active generation/pumping power of the pumped storage unit in all subintervals are determined. In the reactive power optimization, the active generation of all thermal units and active generation/pumping power of the $\mathrm{p}$-s unit in all subintervals are taken as equal to the optimal values that are determined in the active power optimization, only reactive generations of reactive power suppliers are determined. Active and reactive power generation constraints for the unit connected to the swing bus, transmission line capacity constraints, the p-s unit upper reservoir storage constraints, and bus voltage magnitude constraints are added to the solution process via penalty function approach.

The solution method is tested on an example power system, which has 12 buses, 5 thermal units, and one p-s hydraulic unit. The environmental dispatch problem of the example power system without any p-s unit is solved first. After that, the same environmental dispatch problem is resolved for the cases where there is p-s unit with $60 \%, 67 \%$, and $75 \%$ cycle efficiencies, and the obtained results for each case are compared with the corresponding ones that belong to the system without any p-s unit. Savings in the total cost of the system, in the case of employing the p-s unit in the power system, are given for different $w$ values for each $\mathrm{p}$-s unit's cycle efficiency.

The dispatch problem of the example power system with p-s unit is resolved by the F-MSG method, and the decrease percentages on the total cost are recalculated. It is seen that the F-MSG method gives similar but a little bit higher total cost decrease percentages with respect to those produced by the proposed method. The decrease percentages on the total system cost can be increased by performing the active and then reactive optimizations in an iterative manner with the cost of longer solution time.

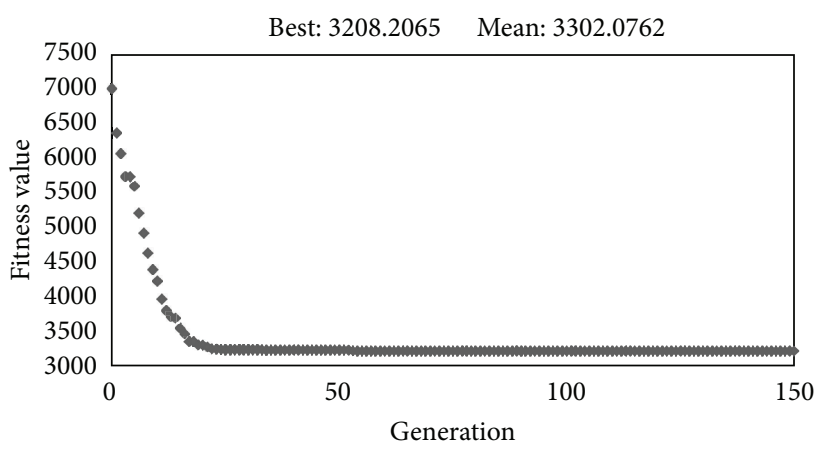

Figure 6: The lowest fitness value calculated versus generation (iteration) number in the reactive power optimization for the case, where there is no p-s unit in the system and $w=0.5$.

The lowest fitness $\left(f^{\mathrm{Q}}\right.$ value in (22)) value calculated versus generation (iteration) number in the reactive power optimization for the case, where there is no $\mathrm{p}$-s unit in the system and $w=0.5$ is shown in the Figure 6 . As it is seen in the figure, the decrease in the lowest fitness value becomes insignificant after the iteration number 25 . If a very sharp solution is not desired, the solution procedure can be stopped at the end of iteration number 25. Therefore the stopping criteria for the considered problem can be stated like this: "if the difference between two consecutive fitness values is less than a predetermined tolerance value and also if this condition is satisfied during $N$ (e.g., $N=10$ ) consecutive iterations, then stop." Selection of this type of stopping criterion other than selecting constant number of generation can reduce the necessary number of iterations (or total solution time) to approximate the optimal solution.

\section{Symbols}

$F_{T}: \quad$ Weighted total system cost $(\mathrm{R})$,

$j, j_{\text {max }}$ : Time interval index and number of time intervals, respectively

$P_{n, j}, P_{G H, j}: \quad$ The $n$th thermal and the p-s hydraulic units' active generation powers in the jth time interval, respectively (MW)

$P_{P H, j}: \quad$ The $\mathrm{p}$-s unit's pumping active power in the $j$ th time interval (pu or MW)

$F_{n}\left(P_{n, j}\right): \quad$ Fuel cost rate of the $n$th thermal unit in the $j$ th time interval $(\mathrm{R} / \mathrm{h})$

$E_{n}\left(P_{n, j}\right): \quad$ Emission rate of the $n$th thermal unit in the $j$ th time interval $(\mathrm{Kg} / \mathrm{h})$

$\xi: \quad$ Emission price $(\mathrm{R} / \mathrm{Kg})$

$w: \quad$ Weighting factor

$t_{j}: \quad$ Length of time interval $j(\mathrm{~h})$

$P_{\text {load }, j}, P_{\text {loss }, j}$ : Total system load (excluding the pumping power of the $\mathrm{p}$-s hydraulic unit if it is operated in pumping mode) and loss in the $j$ th time interval, respectively (pu or MW)

$p_{l}$ : Active power flow on line $l$ (pu or MW) 


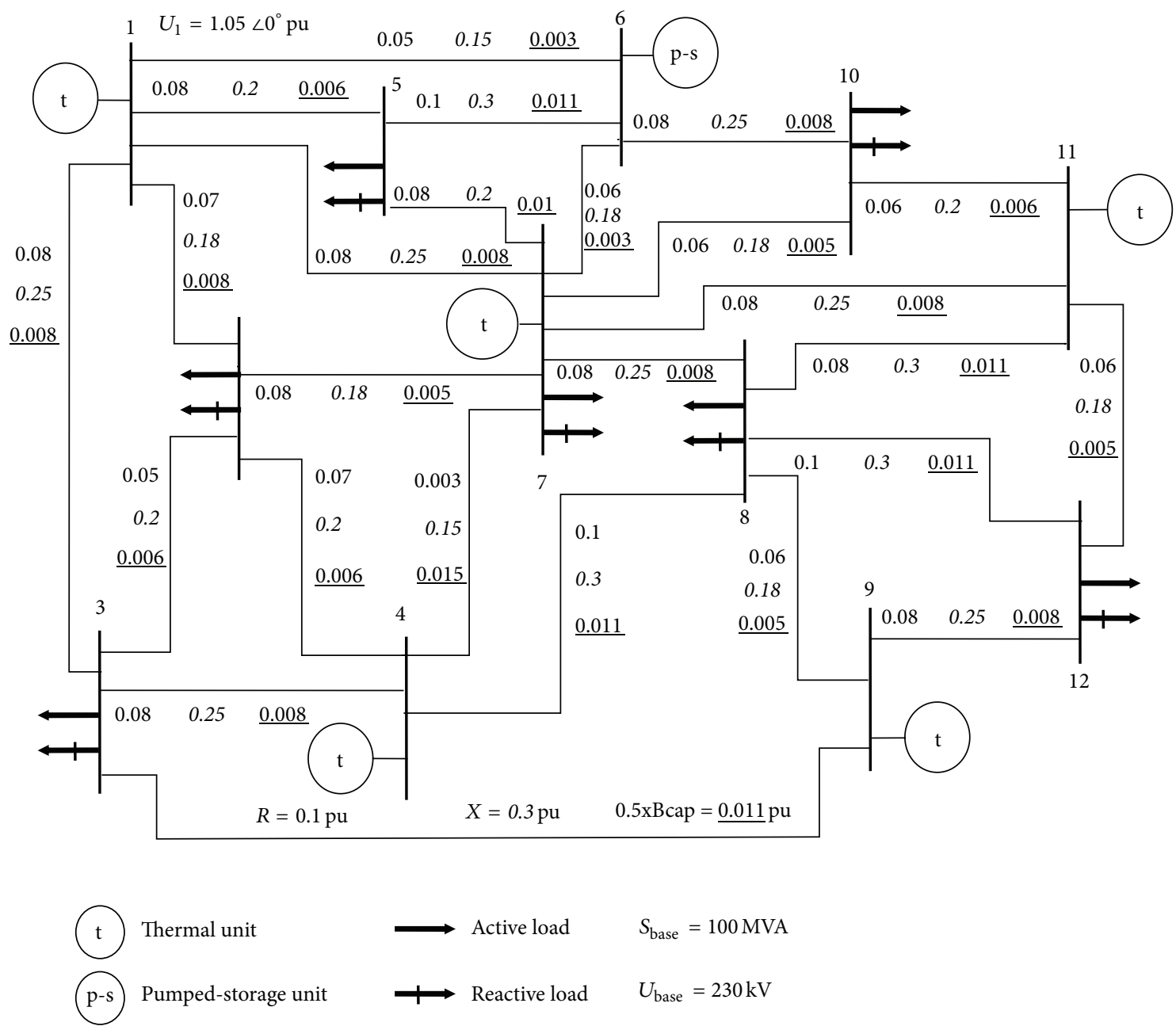

Figure 7: One-line diagram of the example power system.

$p_{l}^{\max }: \quad$ Maximum transmission capacity of transmission line $l$ (pu or MW)

$P_{n}^{\min }, P_{n}^{\max }$ : Lower and upper generation limits of the nth thermal unit (pu or MW)

$P_{G H}^{\min }, P_{G H}^{\max }$ : Lower and upper active generation limits of the p-s hydraulic unit (pu or MW)

$P_{P H}^{\min }, P_{P H}^{\max }:$ Lower and upper active pumping power limits of the p-s hydraulic unit (pu or MW)

$q_{G H}\left(P_{G H, j}\right):$ Discharge rate of the $\mathrm{p}$-s hydraulic unit in the $j$ th time interval $($ acre- $\mathrm{ft} / \mathrm{h})\left(1\right.$ acre- $\left.\mathrm{ft}=1233.5 \mathrm{~m}^{3}\right)$

$q_{P H}\left(P_{P H, j}\right):$ Pumping rate of the p-s hydraulic unit in the $j$ th time interval (acre-ft/h)

$q_{G H}^{\min }, q_{G H}^{\max }: \quad$ Lower and upper discharge rate limits of the p-s hydraulic unit (acre-ft/h)

$q_{P H}^{\min }, q_{P H}^{\max }: \quad$ Lower and upper pumping rate limits of the p-s hydraulic unit (acre-ft/h)
$V_{j}: \quad$ Stored water volume in the upper reservoir of the p-s hydraulic unit at the end of the $j$ th time interval (acre-ft)

$V^{\min }, V^{\max }: \quad \quad$ Minimum and maximum reservoir storage limits of the $\mathrm{p}$-s hydraulic unit (acre-ft)

$q_{\text {net spent }}: \quad \quad$ Net spent water amount by the p-s hydraulic unit during the operation cycle (acre-ft)

$q_{\text {spent ТОT }}, q_{\text {pump ТОт }}$ : Total water amount which is spent for generation and total pumped water amount, respectively (acre-ft)

$V^{\text {start }}, V^{\text {end }}$ : $\quad$ Specified starting and final stored water volumes in the upper reservoir of the p-s hydraulic unit (acre-ft)

$\mathbf{N}_{s}$ : Set that contains all thermal units in a given power system

$\mathbf{N}_{\text {line }}$ : Set that contains all transmission lines in a given power system 


\begin{tabular}{|c|c|}
\hline $\mathbf{J}_{\text {gen }}, \mathbf{J}_{\text {pump }}:$ & $\begin{array}{l}\text { Sets that contain all time intervals } \\
\text { where the p-s unit is operated in } \\
\text { generation and pumping modes, } \\
\text { respectively. } \mathbf{J}_{\text {gen }} \text { and } \mathbf{J}_{\text {pump }} \text { are } \\
\text { mutually exclusive sets }\end{array}$ \\
\hline$F_{\mathrm{TOT}, \mathrm{sw}}:$ & $\begin{array}{l}\text { Weighted total cost for the thermal } \\
\text { unit connected to the swing bus (R) }\end{array}$ \\
\hline$Q_{\text {load }, j}, Q_{\text {loss }, j}:$ & $\begin{array}{l}\text { Total system reactive load and loss in } \\
\text { the } j \text { th subinterval, respectively (pu } \\
\text { or MVAr) }\end{array}$ \\
\hline$Q_{G, m}^{\min }, Q_{G, m}^{\max }$ & $\begin{array}{l}\text { Lower and upper reactive generation } \\
\text { limits of the } m \text { th reactive power } \\
\text { generation source (pu or MVAr) }\end{array}$ \\
\hline$U_{i, j}:$ & $\begin{array}{l}\text { Voltage magnitude of bus } i \text { in the } j \text { th } \\
\text { subinterval ( } \mathrm{kV} \text { or } \mathrm{pu})\end{array}$ \\
\hline$U_{i}^{\min }, U_{i}^{\max }:$ & $\begin{array}{l}\text { Lower and upper voltage magnitude } \\
\text { limits for bus } i(\mathrm{kV} \text { or } \mathrm{pu})\end{array}$ \\
\hline $\mathbf{N}_{\mathrm{Q}}$ : & $\begin{array}{l}\text { Set containing all buses to which } \\
\text { reactive power generation sources are } \\
\text { connected }\end{array}$ \\
\hline $\mathbf{N}_{\text {bus: }}$ : & $\begin{array}{l}\text { Set containing all buses in a given } \\
\text { power system }\end{array}$ \\
\hline $\mathbf{N}_{v c}:$ & $\begin{array}{l}\text { Set containing all voltage controlled } \\
\text { buses in a given power system }\end{array}$ \\
\hline sw: & Swing bus in a given power system. \\
\hline
\end{tabular}

\section{References}

[1] V. N. Dieu and W. Ongsakul, "Enhanced merit order and augmented Lagrange Hopfield network for hydrothermal scheduling," International Journal of Electrical Power and Energy Systems, vol. 30, no. 2, pp. 93-101, 2008.

[2] J. K. Kaldellis, D. Zafirakis, and E. Kondili, "Optimum sizing of photovoltaic-energy storage systems for autonomous small islands," International Journal of Electrical Power and Energy Systems, vol. 32, no. 1, pp. 24-36, 2010.

[3] R. Loisel, "Power system flexibility with electricity storage technologies: A technical-economic assessment of a large-scale storage facility," Electrical Power and Energy Systems, vol. 42, no. 1, pp. 542-552, 2012.

[4] E. Parrilla and J. García-González, "Improving the B\&B search for large-scale hydrothermal weekly scheduling problems," International Journal of Electrical Power and Energy Systems, vol. 28, no. 5, pp. 339-348, 2006.

[5] A. J. Wood and B. F. Wollenberg, Power Generation Operation \& Control, John Wiley \& Sons, New York, NY, USA, 2nd edition, 1996.

[6] S. Fadil and C. Yaşar, "Pseudo spot price algorithm applied to the pumped-storage hydraulic unit scheduling problem," Turkish Journal of Electrical Engineering and Computer Sciences, vol. 8, no. 2, pp. 93-109, 2000.

[7] J. García-González, R. M. R. de la Muela, L. M. Santos, and A. M. Gonzalez, "Stochastic joint optimization of wind generation and pumped-storage units in an electricity market," IEEE Transactions on Power Systems, vol. 23, no. 2, pp. 460-468, 2008.

[8] S. Fadıl and U. Ergün, "Solution to lossy short term hydrothermal coordination problem with limited energy supply thermal units by using genetic algorithm," in Proceedings of the International Conference on Electrical and Electronics Engineering
(ELECO '99), Electrical proceeding, pp. 1234-5238, Bursa, Turkey, December 1999.

[9] D. E. Goldberg, Genetic Algorithms in Search, Optimization, and Machine Learning, Addison-Wesley, Reading, Mass, USA, 1989.

[10] S. Fadil and B. Urazel, "A solution to security constrained nonconvex pumped-storage hydraulic unit scheduling problem by modified subgradient algorithm based on feasible values and pseudo water price," Electric Power Components and System, vol. 41, no. 2, pp. 111-135, 2013. 

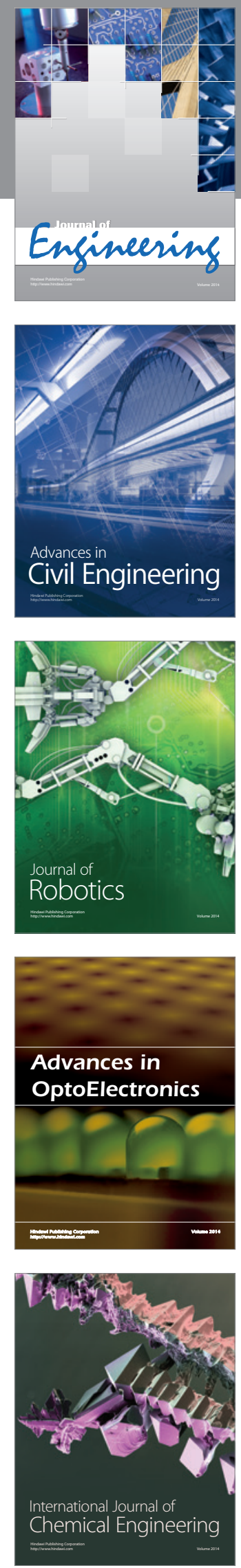

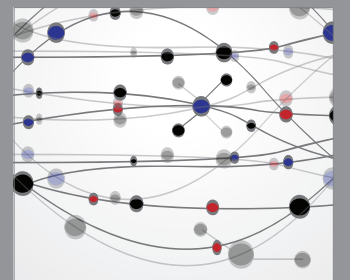

The Scientific World Journal
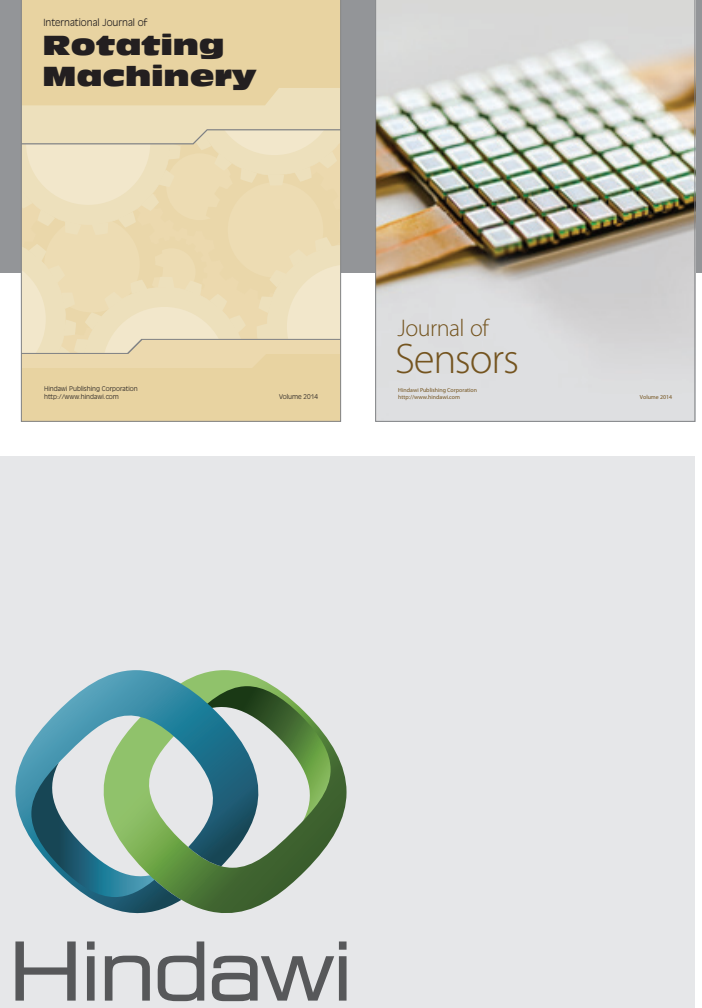

Submit your manuscripts at http://www.hindawi.com

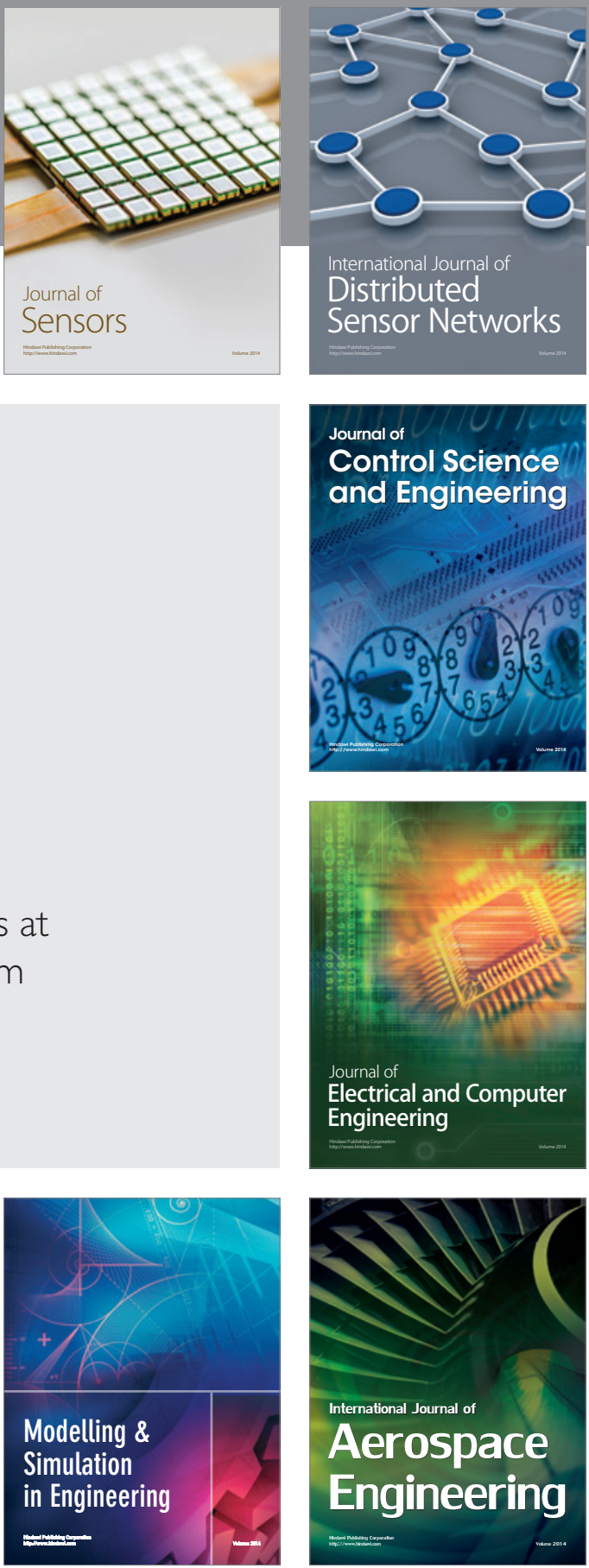

Journal of

Control Science

and Engineering
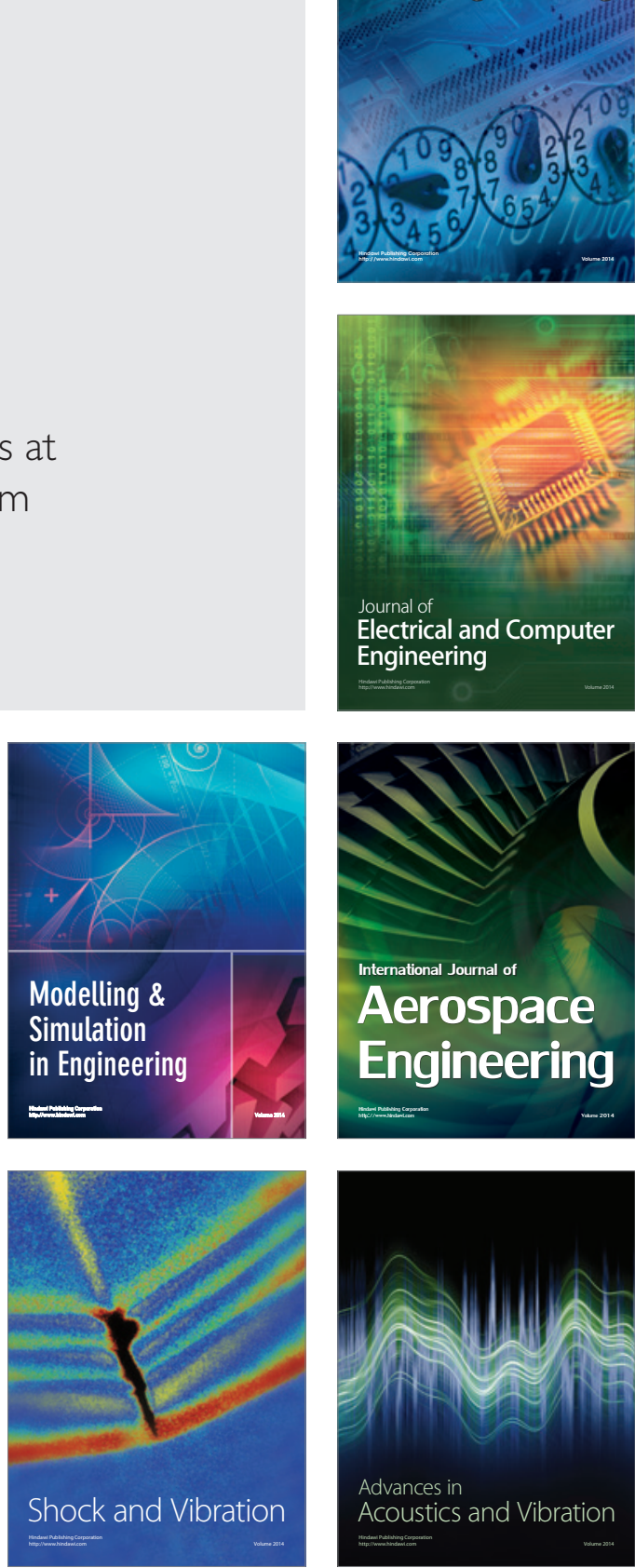